\title{
Echinococcus granulosus Protoscolex DM9 Protein Shows High Potential for Serodiagnosis of Alveolar Echinococcosis
}

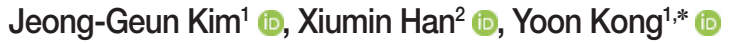 \\ 1 Department of Molecular Parasitology, Samsung Medical Center, Sungkyunkwan University School of Medicine, Suwon 16419, Korea; \\ ${ }^{2}$ Clinical Research Institute of Hydatid Disease, Qinghai Provincial People's Hospital, Xining 810007, China
}

\begin{abstract}
Alveolar echinococcosis (AE) caused by infection with E. multilocularis metacestode, represents one of the most fatal helminthic diseases. AE is principally manifested with infiltrative, proliferating hepatic mass, resembling primary hepatocellular carcinoma. Sometimes metastatic lesions are found in nearby or remote tissue. AE diagnosis largely depends on imaging studies, but atypical findings of imaging features frequently require differential diagnosis from other hepatic lesions. Serological tests may provide further evidence, while obtaining reliable AE materials is not easy. In this study, alternative antigens, specific to AE were identified by analyzing $E$. granulosus protoscolex proteins. An immunoblot analysis of $E$. granulosus protoscolex showed that a group of low-molecular-weight proteins in the range from $14 \mathrm{kDa}$ to $16 \mathrm{kDa}$ exhibited a sensitive and specific immune response to AE patient sera. Partial purification and proteomic analysis indicated that this protein group contained myosin, tubulin polymerization promoting protein, fatty-acid binding protein, uncharacterized DM9, heat shock protein 90 cochaperone tebp P-23, and antigen S. When the serological applicability of recombinant forms of these proteins was assessed using enzyme-linked immunosorbent assay, DM9 protein (rEgDM9) showed $90.1 \%$ sensitivity (73/81 sera tested) and $94.5 \%$ specificity (172/181 sera tested), respectively. $\mathrm{rEgDM9}$ showed weak cross-reactions with patient sera from the transitional and chronic stages of cystic echinococcosis ( 3 to 5 stages). rEgDM9 would serve as a useful alternative antigen for serodiagnosis of both early- and advanced-stage AE cases.
\end{abstract}

Key words: Alveolar echinococcosis, Echinococcus granulosus protoscolex, proteome, specific antigen, serodiagnosis

\section{INTRODUCTION}

Echinococcus multilocularis metacestode infection causes alveolar echinococcosis (AE), a major zoonotic helminthiasis. AE is widely distributed in alpine areas in Europe, Central Asia, northwestern China, the northern part of Japan, and North America [1,2]. Humans are infected by incidental contraction with eggs expelled from canine definitive hosts. When humans become infected with eggs, they are activated in the small intestine (oncosphere), released into the bloodstream, end up lodged in the liver, and grow slowly to form multivesicular cystic masses, in which brood capsules, primary cells, and protoscolices develop [3].

AE lesion is typically manifested with infiltrative hepatic

- Received 2 February 2022, revised 9 February 2022, accepted 10 February 2022.

*Corresponding author (kongy@skku.edu)

(c) 2022, Korean Society for Parasitology and Tropical Medicine

This is an Open Access article distributed under the terms of the Creative Commons Attribution Non-Commercial License (https://creativecommons.org/licenses/by-nc/4.0 which permits unrestricted non-commercial use, distribution, and reproduction in any

medium, provided the original work is properly cited. mass. Sometimes metastatic AE lesions are found in the distinct tissues/organs other than the liver [3]. Patients may complain of abdominal pain, jaundice, hepatomegaly, and weight loss during infection. However, most infections are asymptomatic, usually taking 10 to 15 years after infection, until the mass causes a space-occupying effect or metastatic lesions develop [4]. Patients mostly suffer from non-operable lesions or chronic liver failure when symptoms appear, requiring continuous treatment and monitoring. Early diagnosis and radical treatment may contribute to reducing the disability-adjusted life years and even death [4].

Diagnosis of AE largely depends on a combination of imaging and serological tests [5-7]. However, imaging studies including ultrasonography (US), computed tomography (CT), and/or magnetic resonance (MR) examinations often require a differential diagnosis from other hepatic space-occupying lesions, even for experienced physicians due to their atypical findings. Serological tests may provide additional evidence for $\mathrm{AE}$ [5]. A bundle of proteins was searched for AE serodiagnosis. A recombinant II/3 antigen was isolated by screening $E$. 
multilocularis cDNA library with pooled serum of patients with $\mathrm{AE}$ [8]. Another $54 \mathrm{kDa}$ mucin-containing glycoprotein Em2, localized in the laminated layer of E. multilocularis vesicle, was purified by immunoaffinity chromatography by filtering crossreactive components with cystic echinococcosis (CE) patients' sera $[9,10]$. However, since the immunodominant epitope of Em2 protein is composed of trisaccharides (Gala1-4GalB13GalNAc), widely distributed in several trematodes, the protein caused cross-reaction against sera of other helminthic infections [11]. Another study isolated a $65 \mathrm{kDa} \mathrm{Em} 10$ protein (EmII/3) by cDNA library screening using pooled AE patient sera [12]. Two E. multilocularis protoscolex proteins at $16 \mathrm{kDa}$ and $18 \mathrm{kDa}(\mathrm{Em} 16 / 18)$ were reported to have a sensitivity and specificity of approximately $85 \%$, but these molecules only react with serum samples from active stage AE $[13,14]$. Moreover, these proteins are partial fragments of ezrin-like protein, derived from the ezrin-radexin-moesin family. These proteins bear same epitope, which is hard to detect diverse polyclonal antibodies circulating in infected individuals [15]. The identification and characterization of novel serodiagnostic biomarkers for AE may be beneficial for diagnosing $\mathrm{AE}$ in various clinical settings [16].

In an effort to seek an alternative antigen available for $\mathrm{AE}$, we analyzed diagnostic applicability of $E$. granulosus protoscolex proteins. A protein designated as uncharacterized DM9 of E. granulosus (EgDM9; CDJ22470) was found to have high diagnostic performance. We assessed the diagnostic feasibility of bacterially expressed recombinant EgDM9 (rEgDM9) in serological diagnosis of AE patients.

\section{MATERIALS AND METHODS}

\section{Parasite and serum samples}

E. granulosus protoscolex and hydatid fluid (CE2 stage) were collected from the sheep livers at a slaughterhouse in Xining, Qinghai, China. The E. multilocularis protoscolex was a generous gift from Dr. Wang (Qinghai Centers for Diseases Control and Prevention). The protoscolices were washed with physiological saline containing protease inhibitor cocktail (1 tablet/25 ml, Complete, Roche, Swiss), homogenized with a Teflon-pestle crusher, and centrifuged at 20,000 g for $1 \mathrm{hr}$ at $4^{\circ} \mathrm{C}$. Hydatid fluid was cleared at $500 \mathrm{~g}$ for $2 \mathrm{~min}$, followed by centrifuged at 20,000 g for $1 \mathrm{hr}$ at $4^{\circ} \mathrm{C}$. The supernatants were stored at $-80^{\circ} \mathrm{C}$. Total RNA was isolated from fresh E. granulosus protoscolex using an RNA extraction kit (iNtRON, Seong- nam, Korea). cDNA was reverse transcribed using a first-strand cDNA synthesis kit (Invitrogen, Carlsbad, California, USA).

The sera of patients with $\mathrm{AE}$ and $\mathrm{CE}$ cases stored at Qinghai Provincial People's Hospital was used. The patients were diagnosed based on clinical features and US examination $[17,18]$. We categorized the patients into early and advanced stages according to their US images. The patients whose US could not be reviewed by corresponding author were regarded as stage not-defined. We used $81 \mathrm{AE}$ sera (each of 25 cases of early- and advanced-stage and 31 sera with stage not-defined). A total of 97 sera, which included CE1 (18 sera), CE2 (15 sera), CE3 (21 sera), CE4 (25 cases), and CE5 (18 cases), respectively, was examined. We also tested 33 sera from cysticercosis patients and 51 sera from normal controls, who denied any possible infection to helminths and protozoans. Informed consent was obtained from the patients and doners. The study protocol and use of serum samples were approved by the Institutional Review Board of Qinghai Provincial People's Hospital, Xining, China (2017-7-29).

\section{Electrophoresis and immunoblotting}

For SDS-PAGE, protein samples were separated on 15\% gels under reducing conditions and stained with Coomassie Brilliant Blue G-250 (CBB). Two-dimensional electrophoresis (2DE) was done as previously described [17]. In brief, samples mixed with rehydration buffer were focused on IPG strips $\mathrm{pH}$ 3-10 or pH 4-7 (GE healthcare, Piscataway, New Jersey, USA) and processed with $15 \%$ SDS-PAGE. Proteins separated by SDS-PAGE or 2-DE were electroblotted to nitrocellulose membranes (Santa Cruz, Dallas, Texas, USA). The membranes were blocked with Tris-buffered saline (100 mM, pH 8.0) containing 3\% skim milk and $0.1 \%$ Tween-20 for $1 \mathrm{hr}$. The blots were incubated with individual and/or pooled serum overnight (1:200 or 1:1,000 dilution) at $4^{\circ} \mathrm{C}$ and sequentially with horse radish peroxidase (HRP)-conjugated anti-human IgG (heavyand light-chain specific, Cappel, Solon, Ohio, USA) for $2 \mathrm{hr}$ (1:2,000-1:4,000 dilution). Immunoreactive signals were detected by either 4-chloro-1-naphthol chromogen (4C1N; Sigma-Aldrich, St. Louis, Missouri, USA) supplemented with $0.03 \% \mathrm{H}_{2} \mathrm{O}_{2}$ for 10 min or SuperSignal Chemiluminescence (ECL; GE Healthcare) after 2 min exposure.

\section{Immunoprecipitation}

E. granulosus protoscolex (Egpsc) or E. multilocularis protoscolex (Empsc) (500 $\mu$ g each) was mixed with 10 pooled pa- 
tient serum $(15 \mu \mathrm{l})$ of $\mathrm{AE}, \mathrm{CE}$, or cysticercosis and incubated overnight at $4^{\circ} \mathrm{C}$, after which Protein $\mathrm{A} / \mathrm{G}$ agarose beads $(30 \mu \mathrm{l}$, Santa Cruz) was added, and incubated for $24 \mathrm{hr}$ at $4^{\circ} \mathrm{C}$. The pellet was recovered by brief centrifugation and washed 3 times with wash buffer (20 mM Tris HCl [pH 8.0], $137 \mathrm{mM}$ $\mathrm{NaCl}, 1 \%$ Triton X-100, and $2 \mathrm{mM}$ EDTA). The pellets were separated by $15 \%$ SDS-PAGE and processed to immunoblot probed with pooled serum of AE or CE patient (each $n=10)$.

\section{Column chromatography}

Egpsc proteins $(30 \mathrm{mg} / 1 \mathrm{ml})$ were eluted by a $1.6 \times 80 \mathrm{~cm}$ long Superdex 200 pre-grade fast performance liquid chromatography (FPLC) equilibrated with $20 \mathrm{mM}$ Tris-HCl (pH 8.0) containing $150 \mathrm{mM} \mathrm{NaCl}$ (flow rate: $1 \mathrm{ml} / \mathrm{min}$ ). A total of 80 fractions ( $3 \mathrm{ml} /$ fraction) were collected. The proteins were concentrated using an Amicon Ultra Centrifugal Filter (molecular limit: $3 \mathrm{kDa}$, MilliporeSigma) and analyzed by 15\% SDSPAGE or 2-DE.

\section{Nano-liquid chromatography-electrospray ionization/ multi-stage MS (nano-LC-ESI-MS/MS)}

Protein spots were cut and sliced into fragments from independent gels, reduced with DTT $(10 \mathrm{mM})$, and processed for LC-MS/MS analysis [17]. The analysis was performed using a nanoflow system (Model 1200, Agilent Technologies, Palo Alto, California, USA) connected to a LTQ linear ion trap mass spectrometer (Thermo Electron, San Jose, California, USA). The $12 \mathrm{~cm}$-long reversed phase capillary column with $75 \mathrm{~mm}$ inner diameter and in-house packed with $5 \mu \mathrm{m} 200$ Å poresized Magic C18AQ beads was applied (Michrome BioResources, Auburn, California, USA). The peptides were eluted in a linear gradient from $10 \%$ to $40 \%$ acetonitrile over $65 \mathrm{~min}$. MS survey was scanned for $300-2,000 \mathrm{~m} / \mathrm{z}$ with 3 data-dependent MS/MS scans of isolation width $(1.5 \mathrm{~m} / \mathrm{z})$, normalized collision energy (25\%), and dynamic exclusion duration (180 $\mathrm{sec}$ ). MS data were generated in RAW file format (Thermo Scientific) using the Xcalibur 1.4 with Tune 1.0. Peptide peaks were introduced into MS/MS ions search within a Mascot server (http://www.matrixscience.com/). Mass values were selected using monoisotopic masses. Peptide and MS/MS tolerances were \pm 1.2 and $\pm 0.6 \mathrm{Da}$, respectively. Protein identification of individual ions scores $>43$ was considered significant or demonstrate extensive homology $(P<0.05)$. Cysteine carbamidomethylation and methionine oxidation were considered. MS database search was performed on the NCBI database (http:// www.ncbi.nlm.nih.gov/).

\section{PCR, cloning, and expression of recombinant proteins}

The candidate proteins were searched from nonredundant and expressed sequence tag (EST) against the NCBI database (http://blast.ncbi.nlm.nih.gov/) and gene/protein database of E. granulosus (http://www.gendb.org/Homepage/). Each primer containing BamHI and HindIII restriction sites was prepared (Supplementary Table S1). The genes were amplified from the E. granulosus CDNA library by PCR: 35 cycles for 5 min at $94^{\circ} \mathrm{C}, 45 \mathrm{sec}$ at $94^{\circ} \mathrm{C}, 1 \mathrm{~min}$ at $58^{\circ} \mathrm{C}, 45 \mathrm{sec}$ at $72^{\circ} \mathrm{C}, 1$ $\min$ at $72^{\circ} \mathrm{C}$, with final extension for $10 \mathrm{~min}$ at $72^{\circ} \mathrm{C}$ [19]. The amplicons were purified by QIAquick Gel extraction kit (Qiagen, Hilden, Germany). The plasmids were ligated to pET-28a or pGEX-6p-1 vector and transformed into Escherichia coli DH5a (Takara, Shiga, Japan).

Recombinant plasmids were transformed into E. coli BL21 (DE3) and cultured in the Luria-Bertani medium supplemented with antibiotics $(50 \mu \mathrm{g} / \mathrm{ml})$. Recombinant proteins were induced with $1 \mathrm{mM}$ isopropyl- $\beta$-D-thiogalactopyranoside for 4 $\mathrm{hr}$ at $37^{\circ} \mathrm{C}$. The cells were lysed and centrifuged at $20,000 \mathrm{~g}$ for $1 \mathrm{hr}$. Recombinant proteins were purified using nickel-nitrilotriacetic acid (Ni-NTA) agarose chromatography (Qiagen) or Glutathione Sepharose 4B affinity column (GE healthcare).

\section{Enzyme-linked immunosorbent assay (ELISA)}

After a checkerboard titration, $100 \mu \mathrm{l}$ of EgDM9 $(2.5 \mu \mathrm{g} / \mathrm{ml}$ suspended in $100 \mathrm{mM}$ carbonate-bicarbonate buffer, $\mathrm{pH}$ 9.6) was coated to the wells of a flat-bottom 96-well microplate (Greiner Bio-One, Kremsmuenster, Austria) overnight at $4^{\circ} \mathrm{C}$. One hundred microliters of serum samples (1:200 dilution in phosphate buffered saline containing 0.05\% Tween 20 [PBS/ $\mathrm{T}]$ ) were incubated for $2 \mathrm{hr}$ at $37^{\circ} \mathrm{C}$, subsequently 1:2,000 diluted HRP-conjugated anti-human IgG antibody (100 $\mu \mathrm{l}$, Cappel) was incubated for $2 \mathrm{hr}$ at $37^{\circ} \mathrm{C}$. Color reactions were developed with $100 \mu \mathrm{l}$ of $1 \% 0$-phenylenediamine (Sigma-Aldrich) supplemented with $0.03 \% \mathrm{H}_{2} \mathrm{O}_{2}$ for 20 min in the dark. Reactions were stopped using $2 \mathrm{~N} \mathrm{H}_{2} \mathrm{SO}_{4}(50 \mu \mathrm{l}$, Sigma-Aldrich). The absorbance was measured at $450 \mathrm{~nm}$ on a NEO microplate reader (Biotek, Winooski, Vermont, USA). Sera from healthy donors and PBS/T were used as negative and blank controls, respectively. Results were determined after correction with appropriate blank. 


\section{Statistical analysis}

Positive/negative values were used to normalize the different batches of ELISA results. GraphPad Prism9 software (GraphPad, San Diego, California, USA) and SPSS statistical package (ver25, IBM, Armonk, New York, USA) were used for the analysis of variance. All data are expressed as the mean \pm standard deviation (SD) of at least 3 independent assays. A $P$-value of $<$ 0.05 with a 2-sided hypothesis was employed. Sensitivity, specificity, positive/negative predictive values (PPV/NPV), positive/negative likelihood ratios (PLR/NLR), and 95\% confidence intervals (CIs) were calculated. The receiver operating characteristic (ROC) curve was created by plotting sensitivity versus 1-specificity. The area under the ROC curve (AUC) was used to compare the diagnostic performance pairwise. ROC analysis determined cutoff values defined as the positive and negative values that gave the highest sum of sensitivity and specificity totals.

\section{RESULTS}

\section{Egpsc low-molecular-weight proteins revealed specific} immune responses against $A E$ patient sera

The immunoreactivity profile of E. granulosus and E. multilocularis metacestode proteins was screened against patient sera with echinococcoses. Immunoblot analysis of individual anatomical compartments of E. granulosus and E. multilocularis metacestodes probed with the pooled serum of patients with $\mathrm{AE}$ and $\mathrm{CE}$ revealed that proteins with molecular weights $>25$ $\mathrm{kDa}$ showed a similar reactive pattern against both patient sera (Fig. 1A). However, 3-5 proteins in the $14-16 \mathrm{kDa}$ range exhibited strong antibody responses against AE patient sera, while showing minimal antibody reactions against CE sera (dotted box). The reaction profile of Egpsc and Empsc was further observed by immunoprecipitation. Egpsc proteins immunologically precipitated with the pooled serum of AE patients exhibited specific bands at around $14-16 \mathrm{kDa}$, whereas those from CE or cysticercosis patients revealed negligible reactions. Empsc proteins immnoprecipitated with sera of patients with $\mathrm{AE}$ also showed immunoreactive bands at around $14-16 \mathrm{kDa}$, but their immunoreactive signal was weaker than those of Egpsc (dotted box, Fig. 1B). When the antibody capturing activity was assessed by immunoblotting, this protein group showed overall diagnostic sensitivity and specificity of about $85-90 \%$ in diagnosing patients with $\mathrm{AE}$ (Fig. 1C, D).

\section{Identification of Egpsc low-molecular-weight proteins}

Egpsc low-molecular-weight proteins were enriched using FPLC. Proteins eluted in the fractions 54-58 contained target molecules (gel filtration profile not shown, Fig. 2A). These proteins were separated using 2-DE and processed with immunoblotting probed with pooled serum of patients with $\mathrm{AE}$ and CE. As shown in Fig. 2B, proteins with molecular weights ca. $14-16 \mathrm{kDa}$ with $\mathrm{pI}$ values ranging between $4.5-6.5$ showed specific antibody reactions against the sera of AE patients, but not against $\mathrm{CE}$ sera. This result demonstrated that Egpsc lowmolecular-weight proteins at 14-16 kDa might be good candidates for the serodiagnosis of patients with $\mathrm{AE}$. These spots were eluted from the 2-DE gels, concentrated, and separated by $15 \%$ SDS-PAGE. A broadband between 14 and $16 \mathrm{kDa}$ was observed (Fig. 2C). When this band was subjected to LC-MS/ MS analysis, the proteins were identified to be myosin, tubulin polymerization promoting protein, fatty acid binding protein 2, hsp90 cochaperone P-23, DM9, expressed protein, and antigen S. Fig. 2D summarizes the identification result. Those candidate molecules were procaryotically expressed by generation of recombinant plasmids via PCR amplification. We did not express antigen $S$ and cyclophilin because antigen $S$ was a fragment of E. granulosus endophilin B1, which shows strong antibody responses to patient sera infected with Taeniidae parasites [20]. The cyclophilin has been implicated with leukocyte chemotaxis rather than priming antigen presenting cells [21]. When the immunoreactivity profile of these proteins was evaluated, all recombinant proteins except for EgDM9 did not show specific antibody reactions (Supplementary Fig. S1).

\section{Molecular properties of EgDM9}

The DM9 protein was selected as a target biomarker. Searches in GeneDB (http://www.gendb.org/Homepage/) and GenBank (http://www.ncbi.nlm.nih.gov/) yielded one gene each encoding E. granulosus DM9 (EgrG_000127000, CDJ22470) and E. multilocularis DM9 (Emu__000127000, CDI97494), respectively. Both genes were coded for 139 amino acids. EgDM9 and EmDM9 proteins showed 97.8\% sequence identity each other, while other platyhelminth members revealed 42.4-67.6\% identity. The deduced amino acid sequences of these genes contained $13 \beta$-strand secondary structures based on the Hidden Markov Model. Three mannose-binding residues were identified at Lys116, Try119, and Glu26 [22]. Interestingly, EgDM9 and EmDM9 proteins tightly conserved the same epitope sequences at the same positions (Gly45-Val51, 

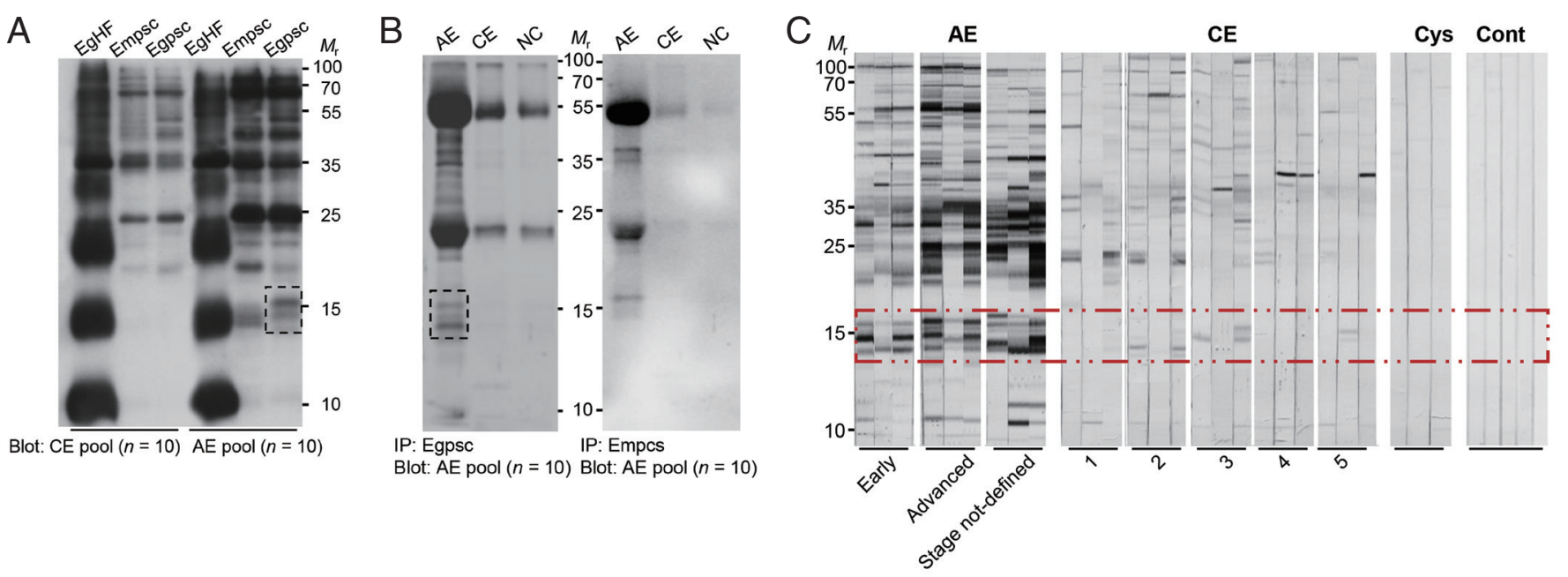

\begin{tabular}{lllll} 
D & & & & \\
\hline Disease category & No. tested & No. positive & \% sensitivity & \% specificity \\
\hline AE & 81 & 74 & 91.4 & - \\
CE & 97 & 15 & - & 84.5 \\
Cysticercosis & 33 & 0 & - & 100 \\
Normal control & 51 & 0 & - & 100 \\
\hline
\end{tabular}

$\mathrm{AE}$, alveolar echinococcosis; $\mathrm{CE}$, cystic echinococcosis

Fig. 1. Identification of immunoreactive proteins of $E$. granulosus protoscolex against patient sera of alveolar echinococcosis (AE) and cystic echinococcosis (CE). (A) Immunoblotting of different anatomical compartments of $E$. granulosus and $E$. multilocularis probed with pooled serum $(n=10)$ of patients with $\mathrm{AE}$ and $\mathrm{CE}$ patients. EgHF, E. granulosus hydatid fluid; Empsc, E. multilocularis protoscolex; EmHF, E. multilocularis hydatid fluid; Egpsc, E. granulosus protoscolex. (B) Immunoprecipitation of Egpsc and Empsc with pooled serum $(n=10)$ of patients with $\mathrm{AE}, \mathrm{CE}$, and cysticercosis. All blots were probed with 1:2,000 diluted pooled serum and 1:4,000 diluted HRPconjugated anti-human IgG antibodies. Immunoreactive signals were detected using ECL after 2-min exposure. (C) Immunoblotting of Egpsc against serum samples from AE, CE, and cysticercosis (Cys), and those from healthy donors (Cont). Dotted box shows a lowmolecular-weight proteins showing specific immunoreactivity. $M_{\mathrm{r}}$, molecular weights in $\mathrm{kDa}$. (D) Assessment of diagnostic performance of low-molecular-weight proteins of Egpsc.

Thr60-Ala67, Ala73-Lys81, and Gly125-Glu130) using Bepipred Linear Epitope Prediction 2.0 analysis (Supplementary Fig. S2).

\section{Diagnostic performance of rEgDM9}

The specific IgG antibody levels of 262 sera from $\mathrm{AE}(n=81)$, CE $(n=97)$, and cysticercosis $(n=33)$ patients were determined, as well as those from healthy controls $(n=51)$ against rEgDM9 with purity greater than $98.5 \%$ using densitometric analysis of SDS-PAGE gel image (inset, Fig. 3A). Serum samples from patients with $\mathrm{AE}$, regardless of disease stages, exhibited significantly higher levels of anti-rEgDM9-specific IgG antibodies compared to other infection groups $(0.44 \pm 0.16$ vs $0.16 \pm 0.04, P<0.0001$, Fig. 3A; Table 1). No significant differences in specific IgG antibody levels against $\mathrm{rEgDM} 9$ were observed between early- and advanced-stage of $\mathrm{AE}(P>0.05)$. The AUC for rEgDM9 was $0.9820 \pm 0.0062$ (Fig. 3B). The ROC curve demonstrated that optimal cutoff abs. of 0.28 distinguished the positive and negative reactions with $95 \%$ Cls of 0.9699-0.9940. PPV and NPV of the test were $88.0 \%$ and $95.5 \%$, respectively. In addition, PLR and NLR were 16.31 and 0.10 , respectively. Table 1 summarized important variables. rEgDM9 showed weak cross-reactions against patient sera from the transitional stage (CE3) and chronic stages (CE4 and CE5), while those from early CE1 and CE2 stages did not show positive responses. Sera from patients with cysticercosis and healthy donors did not react positively (Fig. 3A; Table 1). The diagnostic sensitivity and specificity of rEgDM9-based ELISA were $90.1 \%$ and $94.5 \%$, respectively.

\section{DISCUSSION}

This study investigated the diagnostic feasibility of rEgDM9 using serum samples from hepatic $\mathrm{AE}$ cases. IEgDM9 exhibited 
A

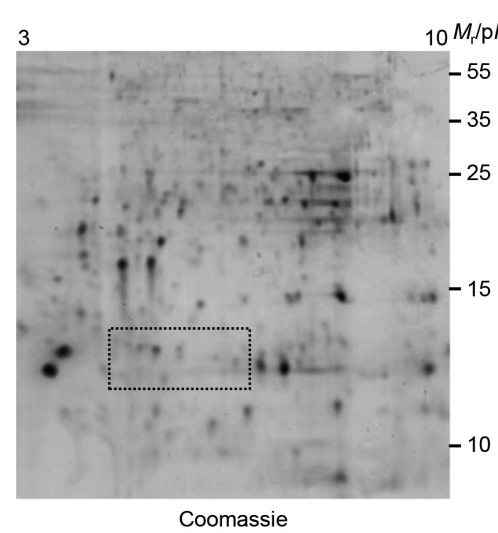

B

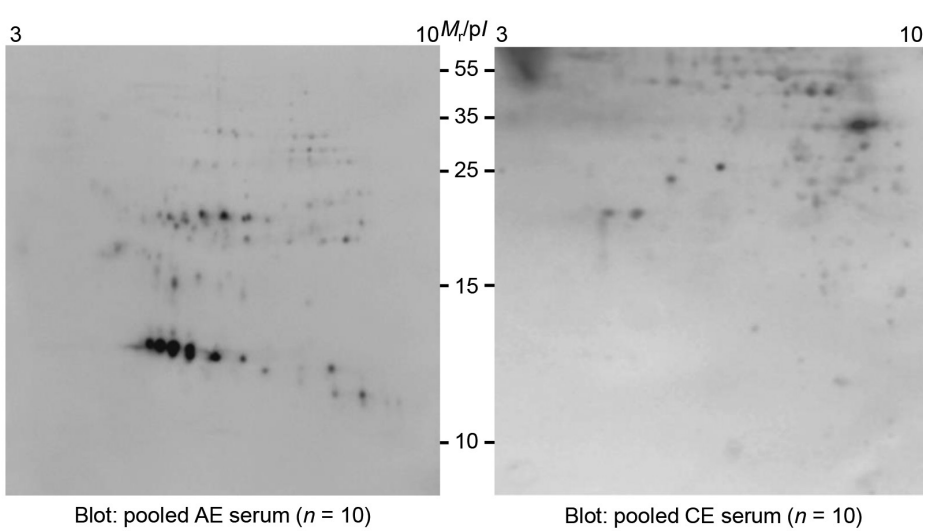

C

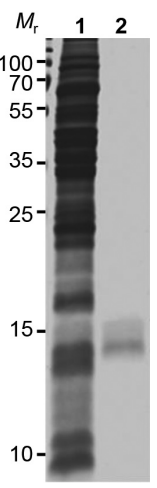

D

\begin{tabular}{llllll}
\hline Entry no. & Mascot score & Matched peptides & Theoretical $M_{\mathrm{r}} / \mathrm{p} /$ & Access. no. & Protein name \\
\hline 1 & 67 & 11 & $23031 / 4.57$ & CDJ18527 & hsp90 cochaperone tebp (P-23) \\
2 & 108 & 8 & $15144 / 5.07$ & CDJ22470 & protein of unknown function DM9 \\
3 & 78 & 12 & $26276 / 5.81$ & CDJ16649 & myosin essential light chain \\
4 & 131 & 12 & $15360 / 5.48$ & AAK12095 & fatty acid binding protein 2 (FABP2) \\
5 & 121 & 1 & $22914 / 5.45$ & CDI70432 & expressed protein \\
6 & 72 & 1 & $17288 / 5.84$ & AAA29056 & antigen S epitope, partial \\
7 & 64 & 10 & $17571 / 6.41$ & P14088 & Cyclophilin (peptidyl-prolyl cis-trans isomerase) \\
\hline
\end{tabular}

Fig. 2. (A) 2-DE profiles of partially purified E. granulosus protoscolex proteins. The proteins eluted through FPLC (fractions 54-59) were pooled, dialyzed, and concentrated. The proteins were electrofocused on the IPGstrip (pH 3-10), separated on 15\% SDS-PAGE, and stained with CBB G-250. Proteins showing specific immunoreactivity against AE patient sera are marked by dotted box. (B) 2-D immunoblots probed with the pooled serum of AE and CE patients $(n=10)$. $M_{r}$, molecular weights in $\mathrm{kDa}$; pl, isoelectric point. (C) SDS-PAGE of partially purified low-molecular-weight proteins. The gels marked by a dotted box in A were sliced and proteins were eluted using EzWay Protein Elution Kit. The proteins were dialyzed, concentrated, and separated by 15\% reducing SDS-PAGE. 1, crude Egpsc; 2 , partially purified proteins. Mr, molecular weights in kDa. (D) Protein identification by LC-MS/MS.

diagnostic sensitivity of $90.1 \%$. Analysis of patient sera infected with phylogenetically closed Taeniidae parasites, such as $E$. granulosus metacestode and Taenia solium metacestode, as well as sera from healthy controls demonstrated an overall specificity of $94.5 \%$. The results suggest that $\mathrm{rEgDM} 9$ has diagnostic potential for serodiagnosis of $\mathrm{AE}$ as an alternative biomarker (Table 1).

The most reliable antigens for $\mathrm{AE}$ serodiagnosis may be derived from the homologous parasite. However, limited parasite resources often hamper obtaining reliable materials in sufficient quantities, especially in AE cases. Several efforts have been made to overcome this shortcoming; E. multilocularis vesicles have been cultured in vitro or infected in severely combined immunodeficient mice $[23,24]$. However, E. multilocularis vesicles obtained under these conditions demonstrated different protein expression profiles compared to those obtained from naturally infected hosts. More importantly, these proteins showed different immune recognition patterns against infection sera $[25,26]$. It remains a challenge to obtain reliable antigens in sufficient amounts available for AE serodiagnosis, and further investigation is needed to identify and isolate a well-defined antigenic molecule that shows reproducible diagnostic performance [13].

In this study, we asked whether Egpsc contained proteins specifically reactive with sera with AE patient because these 2 phylogenetically close cestode parasites shared lots of biochemical and molecular biological singularities $[27,28]$. We observed that the high immunoreactivity of the low-molecular-weight protein group ranged from 14-16 kDa in Egpsc. These molecules were identified using proteomic analysis and recombinant forms of candidate proteins, such as hsp90, EgDM9, myosin, FABP2, and uncharacterized expressed protein were generated. However, the immunoblot experiment of these molecules showed no significant diagnostic applicability other than rEgDM9 (Supplementary Fig. S1). We therefore focused our interest to assess the diagnostic potential of $\mathrm{rDg}$ DM9 using a panel of patient sera infected with Taeniid members. We found that rEgDM9 showed a sensitive and specific 


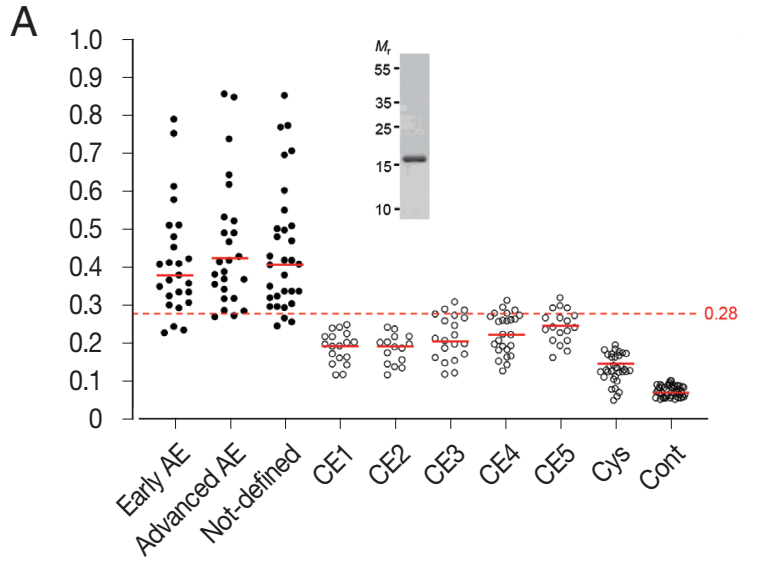

B

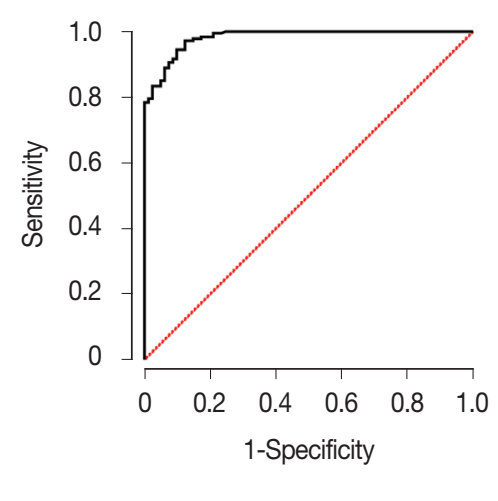

Fig. 3. (A) Specific lgG antibody levels in sera of patients with alveolar echinococcosis (AE), cystic echinococcosis (CE), and cysticercosis (Cyst) against rEgDM9. The $y$-axis shows the absorbance values of the detected sera. The $x$-axis indicates the following sera groups: Early AE, early-stage AE patients whose ultrasonogram demonstrated multiple small hailstorm-like cysts without a solid component $(n=25)$; Advanced AE, advanced-stage AE cases who revealed an irregular cystic area associated with central necrosis surrounded by peripheral non-homogenous echogenicity $(n=25)$; Not-defined, AE patients whose disease stages could not be determined by ultrasonography ( $n=31)$. CE1 $(n=18)$; CE2 $(n=15) ;$ CE3 $(n=21)$; CE4 $(n=25)$; CE5 $(n=18)$; Cys, cysticercosis $(n=33)$; Cont, healthy controls $(n=51)$. Inset demonstrates a homogeneity of tag-cleaved rEgDM9 shown by $15 \%$ reducing SDS-PAGE. Mr, molecular weights in $\mathrm{kDa}$ (B) Analysis of receiver operating characteristic (ROC) curve depicts the plot of sensitivity versus 1 -specificity. The diagonal reference line is also shown. ROC curve was used to determine the cutoff values and area under the curve (AUC) for rEgDM9 in AE serum samples $(n=81)$ by ELISA.

Table 1. Evaluation of recombinant DM9 for serodiagnosis of alveolar echinococcosis

\begin{tabular}{|c|c|c|c|c|c|}
\hline Disease category ${ }^{a}$ & No. of tested & No. of positive & Sensitivity (\%) & Specificity (\%) & Specific antibody levels \\
\hline $\mathrm{AE}$ & 81 & 73 & 90.1 & & $0.44 \pm 0.16$ \\
\hline Early AE & 25 & 22 & 88.0 & & $0.42 \pm 0.14$ \\
\hline Advanced AE & 25 & 23 & 92.0 & & $0.46 \pm 0.17$ \\
\hline Stage not-defined & 31 & 28 & 90.3 & & $0.49 \pm 0.16$ \\
\hline Overall ${ }^{b}$ & 181 & 9 & & 94.5 & $0.16 \pm 0.04$ \\
\hline CE & 97 & 9 & & 89.7 & $0.21 \pm 0.05$ \\
\hline CE1 & 18 & 0 & & 100 & $0.19 \pm 0.04$ \\
\hline CE2 & 15 & 0 & & 100 & $0.18 \pm 0.04$ \\
\hline CE3 & 21 & 3 & & 85.7 & $0.21 \pm 0.06$ \\
\hline CE4 & 25 & 3 & & 88.0 & $0.23 \pm 0.05$ \\
\hline CE5 & 18 & 4 & & 77.8 & $0.25 \pm 0.04$ \\
\hline NC & 33 & 0 & & 100 & $0.15 \pm 0.09$ \\
\hline Healthy controls & 51 & 0 & & 100 & $0.03 \pm 0.01$ \\
\hline PLR & \multicolumn{2}{|c|}{16.31} & & & \\
\hline NLR & \multicolumn{2}{|c|}{0.1} & & & \\
\hline PPV (\%) & \multirow{2}{*}{\multicolumn{2}{|c|}{$\begin{array}{c}88 \\
955\end{array}$}} & & & \\
\hline NPV (\%) & & & & & \\
\hline $\mathrm{AUC}_{ \pm} \mathrm{SE}$ & \multicolumn{2}{|c|}{$0.9820 \pm 0.0062$} & & & \\
\hline $95 \% \mathrm{Cls}$ & \multicolumn{2}{|c|}{0.9699 to 0.9940} & & & \\
\hline Cut-offc & \multicolumn{2}{|c|}{0.28} & & & \\
\hline
\end{tabular}

${ }^{a} \mathrm{AE}$, alveolar echinococcosis; $\mathrm{CE}$, cystic echinococcosis; NC, neurocysticercosis.

boverall specificity was calculated using sera from 130 patients with CE $(n=97)$, NC $(n=33)$ and those from 51 healthy controls.

${ }^{\circ}$ Cut-off values were determined by the analysis of ROC curves.

antibody reactivity against serum samples from AE cases (Fig. 3). Since we were unable to express the recombinant EmDM9 due to unavailability of E. multilocularis cDNAs, we analyzed epitopes shared between EgDM9 and EmDM9 using computer-aided simulation. We recognized that these closely related molecules (sequence identity of $97.8 \%$ ) harbored the same 
epitope sequences at the same regions. This result suggests that EgDM9 and EmDM9 might have common epitopes and that EgDM9 protein can be used as a substitute antigen for EmDM9. The carbohydrate-binding property of DM9 protein might potentiate antibody capturing activity [22] .

Various proteins, lipids, carbohydrates, and small RNAs packaged within extracellular vesicles are secreted into the host. E. granulosus metacestode might directly secrete extracellular vesicles from their syncytial tegument. Some of these proteins released into the host might interact with the host immune system to prime naïve immune cells in the local environment, resulting in production of specific antibodies [2931]. Proteins packaged in extracellular vesicles have been identified among the antigens used for immunodiagnosis of echinococcosis and cysticercosis [32]. We assumed that EgDM9 protein might be secreted as an extracellular vesicle and induced antibody responses. This hypothesis awaits further elucidation.

In this study, we observed that the antigenicity of native EmDM9 protein was not high compared to that of EgDM9 (Fig. 1). We surmise that the low antigenicity might be attributable to the low expression levels of EmDM9. The low antigenicity of native protein due to low expression levels might be significantly increased when a recombinant protein is used. The native form of E. granulosus antigen B2 (EgAgB2) did not exhibit significant antibody responses against the sera of patients with $\mathrm{CE}$, but recombinant EgAgB2 was one of the most reliable antigens for serodiagnosis of CE patients [19,33]. Since E. multilocularis vesicles showed differential expression of diverse proteins during the developmental period [34], expressional regulation of EmDM9 protein should be elucidated in the future.

As CE progressed to chronic stages, the immune responses might be lowered with the regression of the CE cyst [19]. However, it was interestingly observed that patient sera from the transitional and chronic stages of CE cases exhibited cross-reactions with $\mathrm{rEgDM} 9$ to some extent, while $\mathrm{rEgDM} 9$ showed negligible antibody responses against active stage CE sera (Table 1; Fig. 3). Lots of compositional changes occurred within the $\mathrm{CE}$ cyst during involution. When the cysts regressed to CE3-CE5 stages, almost all the hydatid fluids were absorbed, and only degenerative protoscolices remain in the cyst. The antibody responses to several isoforms of antigen $B$, which constituted the major components of hydatid fluid, might be substantially declined. Conversely, specific antibodies against
EgDM9 might be persistently remain to some extent because EgDM9 protein is continuously synthesized in the protoscolex.

In conclusion, $\mathrm{rEgDM} 9$ protein demonstrated a sensitive and specific antibody responses against $\mathrm{AE}$ patient sera. $\mathrm{rEg}$ DM9 would be a good candidate for serodiagnosis of AE cases.

\section{ACKNOWLEDGMENTS}

The authors are grateful to Dr. Wang H, Qinghai Centers for Diseases Control and Prevention for his gift of E. multilocularis protoscolex collected from experimentally infected mouse. This work was supported in part by the Korea Research Foundation (2019R1I1A1AO1058525).

\section{CONFLICT OF INTEREST}

The authors declare no conflict of interest related to this study.

\section{REFERENCES}

1. Eckert J, Conraths FJ, Tackmann K. Echinococcosis: an emerging or re-emerging zoonosis? Int J Parasitol 2000; 30: 1283-1294. https://doi.org/10.1016/s0020-7519(00)00130-2

2. Craig PS, Li T, Qiu J, Zhen R, Wang Q, Giraudoux P, Ito A, Heath D, Warnock B, Schantz P, Yang W. Echinococcosis and Tibetan communities. Emerg Infect Dis 2008; 14: 1674-1675. https:// doi.org/10.3201/eid1410.071636

3. Spiliotis M, Lechner S, Tappe D, Scheller C, Krohne G, Brehm K. Transient transfection of Echinococcus multilocularis primary cells and complete in vitro regeneration of metacestode vesicles. Int J Parasitol 2008; 38: 1025-1039. https://doi.org/10.1016/j.ijpara. 2007.11.002

4. Piarroux M, Piarroux R, Giorgi R, Knapp J, Bardonnet K, Sudre B, Watelet J, Dumortier J, Gérard A, Beytout J, Abergel A, Mantion G, Vuitton DA, Bresson-Hadni S. Clinical features and evolution of alveolar echinococcosis in France from 1982 to 2007: results of a survey in 387 patients. J Hepatol 2011; 55: 1025-1033. https://doi. org/10.1016/j.jhep.2011.02.018

5. Brunetti E, Kern P, Vuitton DA, Writing Panel for the WHO-IWGE. Expert consensus for the diagnosis and treatment of cystic and alveolar echinococcosis in humans. Acta Trop 2010; 114:116. https://doi.org/10.1016/j.actatropica.2009.11.001

6. Kantarci M, Bayraktutan U, Karabulut N, Aydinli B, Ogul H, Yuce I, Calik M, Eren S, Atamanalp SS, Oto A. Alveolar echinococcosis: spectrum of findings at cross-sectional imaging. Radiographics 2012; 32: 2053-2070. https://doi.org/ 10.1148/rg.327125708

7. Bellanger AP, Wang J, Gbaguidi-Haore H, Barrera C, Bresson-Had- 
ni S, Zlobec I, Lachenmayer A, Richou C, Turco C, Gottstein B, Millon L, Beldi G. Investigating new serological and tissue markers for the follow-up of patients operated for alveolar echinococcosis. Parasite Immunol 2021;43: e12827. https://doi.org/10.1111/ pim. 12827

8. Vogel M, Gottstein B, Müller N, SeebeckT. Production of a recombinant antigen of Echinococcus multilocularis with high immunodiagnostic sensitivity and specificity. Mol Biochem Parasitol 1988; 31: 117-125. https://doi.org/10.1016/0166-6851(88)90162-4

9. Deplazes P, Gottstein B. A monoclonal antibody against Echinococcus multilocularis Em2 antigen. Parasitology 1991; 103: 41-49. https://doi.org/10.1017/s0031182000059278

10. Hülsmeier AJ, Gehrig PM, Geyer R, Sack R, Gottstein B, Deplazes $\mathrm{P}$, Köhler P. A major Echinococcus multilocularis antigen is a mucin type glycoprotein. J Biol Chem 2002; 277: 5742-5748. https:// doi.org/10.1074/jbc.M107161200

11. Yamano K, Koizumi A, Takeda T, Kiuchi F, Hada N. Gala1$4 \mathrm{Gal} \beta 1$-3GalNAc is the dominant epitope of Em2 antigen, the mucin-type glycoprotein from Echinococcus multilocularis. Parasitol Res 2012; 111: 795-805. https://doi.org/10.1007/s00436-0122902-1

12. Frosch PM, Frosch M, Pfister T, Schaad V, Bitter-Suermann D. Cloning and characterisation of an immunodominant major surface antigen of Echinococcus multilocularis. Mol Biochem Parasitol 1991; 48: 121-130. https://doi.org/10.1016/0166-6851(91)90108-i

13. Carmena D, Benito A, Eraso E. The immunodiagnosis of Echinococcus multilocularis infection. Clin Microbiol Infect 2007; 13: 460-475. https://doi.org/10.1111/j.1469-0691.2007.01665.x

14. Ishikawa Y, Sako Y, Itoh S, Ohtake T, Kohgo Y, Matsuno T, Ohsaki Y, Miyokawa N, Nakao M, Nakaya K, Ito A. Serological monitoring of progression of alveolar echinococcosis with multiorgan involvement by use of recombinant Em18. J Clin Microbiol 2009; 47: 3191-3196. https://doi.org/10.1128/JCM.01111-09

15. Sako Y, Nakao M, Nakaya K, Yamasaki H, Gottstein B, Lightowers MW, Schantz PM, Ito A. Alveolar echinococcosis: characterization of diagnostic antigen Em18 and serological evaluation of recombinant Em18. J Clin Microbiol 2002; 40: 2760-2765. https://doi.org/10.1128/JCM.40.8.2760-2765.2002

16. Kern P. Clinical features and treatment of alveolar echinococcosis. Curr Opin Infect Dis 2010; 23: 505-512. https://doi.org/10.1097/ QCO.0b013e32833d7516

17. Ahn CS, Cai H, Kim JG, Han X, Ma X, Bae YA, Yang HJ, Kang I, Wang H, Kong Y. An Echinococcus multilocularis antigen B3 proteoform that shows specific antibody responses to active-stage alveolar echinococcosis. J Clin Microbiol 2015; 53: 3310-3317. https://doi.org/10.1128/JCM.01362-15

18. Sulima M, Nahorski W, Gorycki T, Wołyniec W, Wąż P, FelczakKorzybska I, Szostakowska B, Sikorska K. Ultrasound images in hepatic alveolar echinococcosis and clinical stage of the disease. Adv Med Sci 2019; 64: 324-330. https://doi.org/10.1016/j.advms. 2019.04.002

19. Han X, Kim JG, Wang H, Cai H, Ma X, Duong DH, Ahn CS, Kang I, Kong Y. Survey of echinococcoses in southeastern Qing- hai Province, China, and serodiagnostic insights of recombinant Echinococcus granulosus antigen B isoforms. Parasite Vectors 2019; 12: 323. https://doi.org/10.1186/s13071-019-3569-6

20. Ahn CS, Bae YA, Kim SH, Kim JG, Yu JR, Yang HJ, Eom KS, Wang H, Kang I, Yang Y, Kong Y. Spatiotemporal expression patterns and antibody reactivity of Taeniidae endophilin B1. J Clin Microbiol 2016; 54: 2553-2562. https://doi.org/10.1128/JCM. 01135-16

21. Dawar FU, Xiong Y, Khattak MNK, Li J, Lin L, Mei J. Potential role of cyclophilin A in regulating cytokine secretion. J Leukoc Biol 2017; 102: 989-992. https://doi.org/10.1189/jlb.3RU0317090RR

22. Unno H, Matsuyama K, Tsuji Y, Goda S, Hiemori K, Tateno H, Hirabayashi J, Hatakeyama T. Identification, characterization, and X-ray crystallographic analysis of a novel type of mannosespecific lectin CGL1 from the pacific oyster Crassostrea gigas. Sci Rep 2016; 6: 29135. https://doi.org/10.1038/srep29135

23. Nakaya K, Mamuti W, Xiao N, Sato MO, Wandra T, Nakao M, Sako Y, Yamasaki H, Ishikawa Y, Craig PS, Schantz PM, Ito A. Usefulness of severe combined immunodeficiency (scid) and inbred mice for studies of cysticercosis and echinococcosis. Parasitol Int 2006; 55 (suppl): 91-97. https://doi.org/10.1016/j.parint.2005. 11.014

24. Müller N, Frei E, Nuñez S, Gottstein B. Improved serodiagnosis of alveolar echinococcosis of humans using an in vitro-produced Echinococcus multilocularis antigen. Parasitology 2007; 134: 879-888. https://doi.org/10.1017/S0031182006002083

25. Hemphill A, Gottstein B. Immunology and morphology studies on the proliferation of in vitro cultivated Echinococcus multilocularis metacestodes. Parasitol Res 1995; 81: 605-614. https://doi. org/10.1007/BF00932028

26. Matsumoto J, Kouguchi H, Oku Y, Yagi K. Primary alveolar echinococcosis: course of larval development and antibody responses in intermediate host rodents with different genetic backgrounds after oral infection with eggs of Echinococcus multilocularis. Parasitol Int 2010; 59: 435-444. https://doi.org/10.1016/j.parint.2010. 06.003

27. Tsai IJ, Zarowiecki M, Holroyd N, Garciarrubio A, SánchezFlores A, Brooks KL, Tracey A, Bobes RJ, Fragoso G, Sciutto E, Aslett M, Beasley H, Bennett HM, Cai X, Camicia F, Clark R, Cucher M, De Silva N, Day TA, Deplazes P, Estrada K, Fernández C, Holland PWH, Hou J, Hu S, Huckvale T, Hung SS, Kamenetzky L, Keane JA, Kiss F, Koziol U, Lambert O, Liu K, Luo X, Luo Y, Macchiaroli N, Nichol S, Paps J, Parkinson J, PouchkinaStantcheva N, Riddiford N, Rosenzvit M, Salinas G, Wasmuth JD, Zamanian M, Zheng Y; Taenia solium Genome Consortium, Cai J, Soberón X, Olson PD, Laclette JP, Brehm K, Berriman M. The genomes of four tapeworm species reveal adaptations to parasitism. Nature 2013; 496: 57-63. https://doi.org/10.1038/ nature 12031

28. Ahn CS, Kim JG, Han X, Kang I, Kong Y. Comparison of Echinococcus multilocularis and Echinococcus granulosus hydatid fluid proteome provides molecular strategies for specialized host-parasite 
interactions. Oncotarget 2017; 8: 97009-97024. https://doi. org/10.18632/oncotarget.20761

29. Twu O, Johnson PJ. Parasite extracellular vesicles: mediators of intercellular communication. PLoS Pathog 2014; 10: e1004289. https://doi.org/10.1371/journal.ppat.1004289

30. Nicolao MC, Rodriguez Rodrigues C, Cumino AC. Extracellular vesicles from Echinococcus granulosus larval stage: isolation, characterization and uptake by dendritic cells. PLoS Negl Trop Dis 2019; 13: e0007032. https://doi.org/10.1371/journal.pntd.0007032

31. Drurey C, Maizels RM. Helminth extracellular vesicles: interactions with the host immune system. Mol Immunol 2021; 137: 124-133. https://doi.org/10.1016/j.molimm.2021.06.017

32. Ancarola ME, Marcilla A, Herz M, Macchiaroli N, Pérez M, Asurmendi S, Brehm K, Poncini C, Rosenzvit M, Cucher M. Cestode parasites release extracellular vesicles with microRNAs and immunodiagnostic protein cargo. Int J Parasitol 2017; 47: 675-686. https://doi.org/10.1016/j.ijpara.2017.05.003

33. Hernández-González A, Santivañez S, García HH, Rodríguez S, Muñoz S, Ramos G, Orduña A, Siles-Lucas M. Improved serodiagnosis of cystic echinococcosis using the new recombinant 2B2t antigen. PLoS Negl Trop Dis 2012; 6: e1714. https://doi.org/10. 1371/journal.pntd.0001714

34. Ahn CS, Kim JG, Han X, Bae YA, Park WJ, Kang I, Wang H, Kong Y. Biochemical characterization of Echinococcus multilocularis antigen B3 reveals insight into adaptation and maintenance of parasitic homeostasis at the host-parasite interface. J Proteome Res 2017; 16: 806-823. https://doi.org/10.1021/acs.jproteome.6b00799 